\title{
Author Correction to: Biotransformation of Ginsenoside Rb1 to Ginsenoside CK by Strain XD101: a Safe Bioconversion Strategy
}

\author{
Yunyun Jiang ${ }^{1} \cdot$ Weina $\mathrm{Li}^{2} \cdot$ Daidi Fan ${ }^{3}$ \\ Published online: 22 April 2021 \\ C) Springer Science+Business Media, LLC, part of Springer Nature 2021
}

\section{Author Correction: Applied Biochemistry and Biotechnology https://doi.org/10.1007/s12010-021-03485-0}

The original version of this article unfortunately contained a mistake in the affiliation, authors' contribution and funding. The correct data are shown here.

The original article has been corrected.

Author contribution JYY, LWN, and FDD conceived and designed the study. JYY performed the experiments and analyzed the data. JYY wrote the paper. LWN and FDD reviewed and edited the manuscript. All the authors read and approved the manuscript.

Funding This research was supported by the National Natural Science Foundation of China (21706211, 21576160, 21878246, 21676214), the National Key R\&D Program of China (2019YFA0905200), and the Educational Commission of Shaanxi Province of China (16JS104).

Publisher's Note Springer Nature remains neutral with regard to jurisdictional claims in published maps and institutional affiliations.

JYY and LWN contributed equally to this work.

The online version of the original article can be found at https://doi.org/10.1007/s12010-021-03485-0

\author{
Daidi Fan \\ fandaidi@nwu.edu.cn \\ Yunyun Jiang \\ m15739511396@163.com \\ Weina $\mathrm{Li}$ \\ zhencheng874125@163.com
}

1 Shaanxi Key Laboratory of Degradable Biomedical Materials, School of Chemical Engineering, Northwest University, Taibai North Road 229, Xi'an 710069, China

2 Shaanxi R\&D Center of Biomaterials and Fermentation Engineering, School of Chemical Engineering, Northwest University, Xi'an 710069, China

3 Biotech \& Biomed Research Institute, Northwest University, Taibai North Road 229, Xi'an 710069 Shaanxi, China 